Placenta. 2019 June ; 81: 42-45. doi:10.1016/j.placenta.2019.04.004.

\title{
Post Traumatic Stress Disorder (PTSD): the psychological sequelae of Abnormally Invasive Placenta (AIP)
}

\author{
Isabel D TOL ${ }^{1}$, Michael YOUSIF ${ }^{2}$, and Sally L. COLLINS ${ }^{3,4}$ \\ Oxford, United Kingdom \\ ${ }^{1}$ The Medical Sciences Division, University of Oxford, Oxford, UK \\ 2Department of Psychological Medicine, Oxford University Hospitals, Oxford, UK \\ ${ }^{3}$ Nuffield Department of Obstetrics and Gynaecology, University of Oxford, UK \\ ${ }^{4}$ The Fetal Medicine Unit, John Radcliffe Hospital, Oxford, UK
}

\begin{abstract}
Introduction-Abnormally invasive placenta (AIP) is a rare pregnancy complication often resulting in postpartum haemorrhage ( $\mathrm{PPH}$ ) and emergency peripartum hysterectomy (EPH). The risk of developing post traumatic stress disorder (PTSD) following unexpectedly traumatic childbirth is known however there is no evidence regarding PTSD in AIP. This pilot study assesses the risk of PTSD for women with AIP compared to women having an uncomplicated caesarean delivery (CD) or unexpected PPH or EPH.
\end{abstract}

Methods-Retrospective case-controlled questionnaire study in a UK Tertiary obstetric unit. Women with AIP (Group-1) were matched by delivery date to control groups: Group-2, women with an uncomplicated CD; Group-3 women referred to a specialist clinic for suspected AIP, but had a normal placenta and uncomplicated CD; Group-4, women who had an unexpected EPH and/or severe (>3000mls) PPH. 218 women were sent a validated PTSD screening questionnaire (Impacts of Events Scale-Revised [IES-R]).

Results—Likelihood of PTSD was recorded for 69 women who responded, revealing significantly higher PTSD scores for women with AIP compared to uncomplicated CD $(P=0.001)$. No significant difference was seen between AIP and EPH/PPH $(P=0.89)$. The number of women with scores high enough to indicate probable PTSD was significantly greater with AIP than uncomplicated $\mathrm{CD}$ group $(P=0.045)$.

Discussion-This study demonstrates that women antenatally diagnosed with AIP and anticipating a potentially traumatic delivery, are at significantly increased risk of developing PTSD. Improved awareness of the negative psychological impact of AIP may increase the number of women being identified and treated, thereby improving their quality of life.

\section{Keywords}

Abnormally invasive placenta; Placenta Accreta; Post traumatic stress disorder; Postnatal mental health 


\section{Introduction}

Abnormally invasive placenta (AIP: also called placenta accreta spectrum) is a rare obstetric complication affecting around 1-3 in every 10,000 births ${ }_{1}$ in Europe but the incidence has been reported to be higher in other countries including the USA. It is the leading cause of caesarean hysterectomy in the developed world 2 with the average blood loss being reported to be as high as 3 to 5 litres.3 The predominant risk factor is previous caesarean delivery (CD), a procedure that has dramatically increased in frequency over the past 50 years. 4,5 Caesarean birth rates are projected to rise further in the future, 6 with the prevalence of AIP likely to follow suit. 7 It is therefore important that clinicians understand not only how to manage the condition to reduce physical morbidity but also the potential long-term psychological consequences for women affected.

Adverse psychological outcomes including post-traumatic stress disorder (PTSD) have been reported as a result of traumatic birth events including severe postpartum haemorrhage $(\mathrm{PPH})$ and emergency postpartum hysterectomy (EPH). ${ }_{8}$ A recent report from France demonstrated that $64 \%$ of women who underwent an unplanned hysterectomy at the time of delivery suffered from PTSD.9 A study published in $2011,{ }_{10}$ showed that $76 \%$ of women who had suffered severe PPH during birth experienced negative memories following delivery, with $41 \%$ reporting persistent problems. Of these individuals, $57 \%$ thought about the delivery for at least a month after, $18 \%$ had an unshakeable fear of dying and $11 \%$ said that the event played at least some part in relationship difficulties or divorce. This demonstrates that both significant blood loss and hysterectomy at delivery, as seen with AIP, can contribute to a number of negative psychological responses. Furthermore, narratives from women experiencing EPH report grief for the loss of the uterus and degradation of feminine identity. 11

These findings, however, may not be directly applicable to women with AIP as unlike many of the participants of the published studies, women with an antenatal diagnosis of AIP are prepared for planned hysterectomy with the possibility of severe PPH and are usually delivered electively by a senior obstetrician and experienced multi-disciplinary team. What is unknown is whether antenatal anticipation of these outcomes influences the risk of developing PTSD as a result of the delivery. Anecdotal evidence from informal questioning indicated that some women with AIP were suffering significant psychological trauma but the true extent of the problem remains unknown.

This pilot study aimed to assess the psychological impact, with particular reference to PTSD, of the diagnosis and treatment for AIP in comparison to women experiencing an uncomplicated CD; and whether these effects are comparable to those seen with unexpectedly traumatic birth experiences.

\section{Materials and methods}

Women were eligible to take part if they were over the age of 16, willing and able to give informed consent for participation and fluent in English. Women who had given birth between September 2012 and September 2016 were eligible for inclusion. 
The study group (Group 1; AIP) consisted of women who were referred to the specialist placenta clinic and diagnosed with AIP on ultrasound, which was confirmed at elective delivery using the FIGO intra-operative grading scheme ${ }_{12}$. They were treated with either caesarean hysterectomy or conservative management (hysterotomy to deliver the baby then uterine closure with the placenta left in situ and follow up until the placenta had reabsorbed). The controls consisted of women who delivered in the same obstetric unit.

Group 2 (CD) was women who were referred for $\mathrm{CD}$ due to a previous $\mathrm{CD}$ and who experienced an uncomplicated CD with an estimated blood loss (EBL) of <1L. Women were identified using the theatre log books. Details of the delivery were then obtained from the hospital electronic records system to check for any complications and confirm EBL and also to ensure they had not attended the specialist placenta clinic.

Group 3 (Clinic+CD) consisted of women who were referred to the specialist placenta clinic with risk factors for AIP however, following an ultrasound scan, were cleared of the diagnosis. These women were only included if they were subsequently delivered by an uncomplicated caesarean with an EBL $<1 \mathrm{~L}$. Participants were identified from the placenta clinic records and their delivery information was checked on the hospital records to confirm an uncomplicated $\mathrm{CD}$.

Group 4 (EPH/PPH) consisted of women who experienced unexpected severe PPH (EBL $>3 \mathrm{~L}$ requiring transfer to theatre) and/or an unanticipated EPH. All severe PPH and EPH cases generate a clinical governance form, which is then held on a hospital database. This database was used to identify women potentially meeting this criteria then the full details of their delivery were checked from their hospital records to confirm that there had been no antenatal reason to anticipate an increased risk of a complicated or traumatic birth.

All controls were case matched for date of delivery (+/- 3 months) with each AIP case in an attempt to minimise the confounding effect of differential recall occurring due to time since delivery. For every AIP participant $(n=35)$, we endeavoured to identify two women in each control group (group 2, n=64; Group 3, n=53; Group 4, n=60) in an attempt to account for the low response rate usually seen with postal questionnaire studies and to increase the sample size and subsequent power of the study. The hospital electronic records of all potential participants were checked to ensure that there was no record of neonatal mortality or significant morbidity. This was to prevent confounding of the study results and avoid unnecessary distress to participants.

All potential participants were sent an invitation letter specific to their cohort (Document S1), a patient information sheet (Document S2), two consent forms (Document S3) and, a validated PTSD screen questionnaire (Document S4).

If no response was received within two weeks, a second set of documents were sent. If there was no reply to this no further contact was attempted to comply with the instructions of the local ethics committee. To mitigate poor response rates, 13 questionnaires and letters were personalised; a stamped return envelope was included; packs were sent by first class post; and a second copy of documents along with a reminder letter were sent 2 weeks after the first pack. 
Throughout the study, all women had the right to withdraw at any time, without giving a reason. Data was assessed within 2 weeks of receipt and individuals that screened positive for PTSD were informed and their GP was contacted so that a mental healthcare referral could be pursued where indicated. If women screened negative they were not contacted again.

The short, validated PTSD screening questionnaire, impact of events scale-revised (IES-R), 14 was used to indicate whether or not the woman was likely to be suffering from PTSD. This tool is a 22 item self-reporting questionnaire that can be used to identify increased probability of PTSD diagnosis. It takes around 5 minutes to complete. Scores range from 0-88; below 24 indicates a low PTSD risk, 24-32 suggests PTSD is a clinical concern, 33 and above implies probable PTSD. 14

\section{Statistical Analyses}

The data was tested for normal distribution using Kolmogorov-Smirnov comparison test. Non-parametric tests (Mann Whitney U and Fisher's exact test) were used where the data were not normally distributed. Statistical significance was assumed at a p value of 0.05 or less.

\section{Ethical approval}

The study was undertaken following ethical review obtained from South Central - Oxford B Research Ethics Committee on 11/11/2016 (16/SC/0516).

\section{Results}

Of the 218 women contacted, 69 replied (32\% response rate): 17 out of 35 in AIP (49\% response rate), 14 out of 66 in $\mathrm{CD}(21 \%), 16$ out of 53 in Clinic+CD (31\%) and 22 out of 65 in EPH/PPH (34\%). Of the women with AIP who replied, 16 had an immediate hysterectomy and 1 had successful conservative management. All had histopathological confirmation of AIP and a clinical confirmation of an AIP FIGO grade 4 or higher. Of the 22 women in the EPH/PPH group all had an unexpected blood loss of greater than $3000 \mathrm{mls}$ and were transferred to the operating theatre but all were managed conservatively with none having a hysterectomy.

Table 1 shows the time since delivery for all women that returned questionnaires. There was no significant difference in time since delivery between the AIP cohort and the other categories.

The PTSD screening scores for the EPH/PPH group were normally distributed but the scores for the three other groups were not, therefore non-parametric tests were used (MannWhitney U and Fisher's exact test). The median PTSD screening scores for women with confirmed AIP and unexpected EPH/PPH (Table 2) were significantly higher than those of women who had an uncomplicated CD ( $P=0.001)$. The median PTSD screening scores for women who attended the specialist placenta clinic for risk of AIP but went on to have an uncomplicated $\mathrm{CD}(\mathrm{Clinic}+\mathrm{CD})$ were not significantly different to women who were not at risk of AIP and just had an uncomplicated $\mathrm{CD}(P=0.726)$ (Table 2). No significant 
difference was seen between the median IES-R scores for women with AIP when compared to women who had an unexpected EPH/PPH $(P=0.89)$.

A score of $>32$ on the IES-R represents the cut-off for probable PTSD .15 The number of women scoring for probable PTSD was significantly greater in the AIP cohort $(n=7)$ than in the uncomplicated $\mathrm{CD}$ group $(\mathrm{n}=1, P=0.045)$. However, a significant difference was not seen in the number of women with probable PTSD between the uncomplicated CD and unexpected EPH/PPH group $(\mathrm{n}=7, P=0.12)$ or clinic $+\mathrm{CD}$ group $(\mathrm{n}=0,\{P=0.47)$.

\section{Discussion}

An unexpectedly traumatic birth experience has been shown to be associated with an increased risk of developing PTSD. ${ }_{11,12}$ However, this is the first study to demonstrate that women who are antenatally diagnosed with AIP and are therefore anticipating a difficult, potentially traumatic delivery, are still at significantly increased risk of developing PTSD.

PTSD is associated with a wide range of traumatic events including childbirth, where an individual feels that their life is at risk. The neurobiology underpinning this disorder remains unclear. Research has suggested that symptoms may arise as a result of increased CSF catecholamine secretion, 16 which can trigger the formation of neurological patterns in the brain. Along with hypersensitivity of the amygdala 17 and hypoactivity of the anterior cingulated cortex, ${ }_{17}$ this is thought to increase the likelihood of an individual reacting disproportionately to subsequent fearful situations. AIP is associated with PPH and peripartum hysterectomy; critical events that can be experienced as traumatic. Our findings indicate that being prepared for the possibility of these life threatening circumstances does not necessarily mitigate the risk of developing PTSD postnatally. Early detection of the psychological sequelae of AIP through a systematic screening program might enable prompt intervention by facilitating access to psychological and mental health services for women developing PTSD.

Some women also returned narrative comments with the questionnaires, reporting difficulties with intra-familial relationships alongside PTSD symptoms. One woman stated "my birth experience has affected many relationships and so many aspects of my life. People don't recognise what I have lost as I have my son”. Others revealed that they felt that their healthcare professionals outside of the hospital setting, friends and family did not understand AIP and so were unable to empathise. "To go from amazing care to no contact with people who understood was hard and resulted in me shutting it all out too soon I think" Many also conveyed that they felt a peer support group would have been beneficial for their recovery. This demonstrates that approaches to addressing the negative psychological impact might include increasing awareness of AIP and postnatal psychological risks among universal healthcare professionals (GPs, midwives, health visitors) as they are most likely to be the healthcare professionals in contact with the women.

The rarity of this condition means a small sample size was unavoidable. It was impossible to perform a power calculation as there was no effect size estimate available in the literature. Therefore, sample size was based on the maximum number of women available from the 
database of women who had been diagnosed and treated with AIP since the specialist service had been set up. Despite the low numbers however, the results were statistically significant in support of the proposed hypothesis demonstrating that there is likely to be a sizeable effect at play and that the study was probably not underpowered for the simple question being investigated.

A prospective approach would be the 'gold standard' for this study, although it would not necessarily completely eliminate recollection bias. However, the time period required to prospectively recruit this number of women in a single centre would be several years. Therefore, a retrospective case-control study was the most practical design available for this pilot study. To mitigate recollection bias, we attempted to case-control by delivery date. The results demonstrate that there was no significant difference between the number of responses from women with AIP and those from the other groups for any of the time periods indicating that the case matching for time since delivery was reasonable. Studies such as this are also believed to suffer selection bias, as those negatively affected by their experience are more likely to respond which may explain the possibly surprising finding of a women in the uncomplicated caesarean delivery group who reported symptoms of severe PTSD.

'Cold' postal questionnaires are renowned for poor responses, usually in single figures. To mitigate this we employed a number of modifications that have been found to increase response 14 which may explain the relatively high $32 \%$ return rate in this study.

As the study covered a period of 4 years there is the potential for bias due to the MDT gaining more experience in managing AIP. However, we believe that this bias will be minimal as the members of the MDT did not change and whilst our management is always personalised to the unique circumstances of each woman, the mainstay of our treatment has always been hysterectomy with only a small number opting for expectant management."

\section{Conclusion}

Caesarean section rates are rising and therefore cases of AIP are set to follow suit. Our study has revealed that this could be associated with an increased prevalence of women suffering from child-birth related PTSD. Currently there are no evidence based interventions available to prevent PTSD. ${ }_{17}$ Contemporary understanding of the condition indicates that post-event intervention should be the primary focus. Therefore, healthcare professionals, especially those involved in postnatal care of women, need to be educated about AIP and its potential negative psychological impact so that these women at high risk of developing PTSD can be identified early and access appropriate treatment.

\section{Acknowledgements and funding}

Miss Isabel Tol is funded by the Medical Sciences Department, University of Oxford. Prof. Sally Collins is supported by the Eunice Kennedy Shriver National Institute of Child Health and Human Development (NICHD) Human Placenta Project of the National Institutes of Health under award number UO1 HD 087209. Funders did not contribute to study design, collection or analysis. 


\author{
Abbreviations: \\ PTSD - \\ AIP - \\ CD - \\ EPH - \\ PPH - \\ EBL - \\ IES-R - \\ post traumatic stress disorder \\ abnormally invasive placenta/placenta accreta \\ caesarean delivery \\ emergency postpartum hysterectomy \\ postpartum haemorrhage \\ estimated blood loss \\ impact of events scale-revised
}

\title{
References
}

1. Thurn L, Lindqvist PG, Jackobsson. Abnormally invasive placenta-prevalence, risk factors and antenatal suspicion: results from a large population-based pregnancy cohort study in the Nordic countries. BJOG 2013;123(8):1348-55.

2. Shellhaas C, Gilbert S, Landon M. The frequency and complication rates of hysterectomy accompanying cesarean delivery. Obstetrics and Gynecology 2009;114(2, Part 1):224-229. [PubMed: 19622981]

3. Hudon L, Belfort MA, Broome DR Diagnosis and management of placenta percreta: a review. Obstet Gynecol Surv 1998;53:509. [PubMed: 9702791]

4. Taffel S, Placek P, Liss T. Trends in the US caesarean section rate and reasons for the 1980-85 rise. Am J Public Health 1987;77(8):955-959. [PubMed: 3605474]

5. Martin J, Hamilton B, Osterman M. Births in the US. NCHS Data Brief 2014; 216(3)

6. Mitteroecker P, Huttegger S, Fischer B, Pavllcev M. Cliff-edge model of obstetric selection in humans. PNAS 2016;113(51):14680-14685. [PubMed: 27930310]

7. Guleria K, Gupta B, Agarwal S, Suneja A, Vaid N, Sandhya J. Abnormally invasive placenta: changing trends in diagnosis and management. Acta Obstetricia et Gynecologica Scandinavica 2013;92(4):461-464. [PubMed: 23517217]

8. Howard L Molyneaux E Milgrom J Non-psychotic mental disorders in the perinatal period. Lancet 2014;384(9956):1775-1788. [PubMed: 25455248]

9. Sentilhes L, Gromez A, Clavier E, Resch B, Descamps P, Marpeau L. Long-term psychological impact of severe postpartum hemorrhage. Acta Obstetricia et Gynecologica Scandinavica 2011;90(6):615-620. [PubMed: 21370999]

10. Michelet D, Ricbourg A, Gosme C, Rossignol M. Emergency hysterectomy for life-threatening postpartum haemorrhage: Risk factors and psychological impact. Gynécologie, obstétrique et fertilité 2015;43(12):773-779.

11. Wolf S Emotional reactions to hysterectomy. Postgrad Med 1970;47(5):165-9.

12. Jauniaux E, Chantraine F, Silver R and Langhoff-Roos J FIGO consensus guidelines on placenta accreta spectrum disorders: Epidemiology. International Journal of Gynecology \& Obstetrics 2018; 140(3):265-273. [PubMed: 29405321]

13. Edwards P, Roberts I, Clarke M. Increasing response rates to postal questionnaires: systematic review. BMJ 2002;324:1183. [PubMed: 12016181]

14. Sundin E \& Horowitz M Impact of Event Scale: Psychometric properties. British Journal of Psychiatry 2002;180:205-9. [PubMed: 11872511]

15. Geracioti T et al. CSF Norepinephrine Concentrations in Posttraumatic Stress Disorder. American Journal of Psychiatry 2001;158: 1227-1230. [PubMed: 11481155] 
16. Etkin A \& Wager T Functional Neuroimaging of Anxiety: A Meta-Analysis of Emotional Processing in PTSD, Social Anxiety Disorder, and Specific Phobia. American Journal of Psychiatry 2007;164;1476-1488. [PubMed: 17898336]

17. Gartlehner G, Forneris CA, Brownley K. Interventions for the Prevention of Posttraumatic Stress Disorder (PTSD) in Adults After Exposure to Psychological Trauma. Comparative effectiveness review 2013;109. 


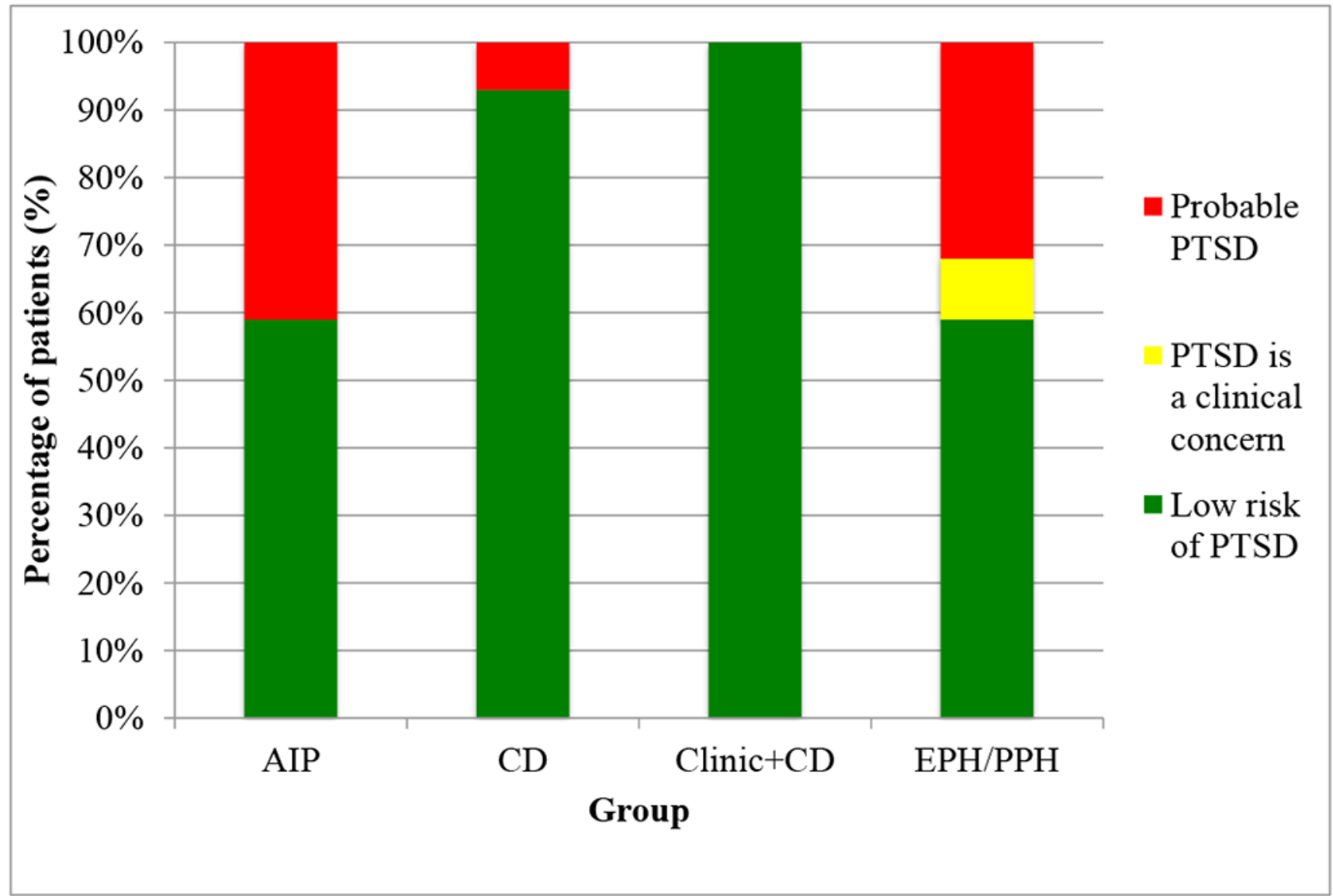

Figure 1.

Percentage of women in each risk category according to their IES-R score 


\section{Table 1}

Time since delivery for all women that returned questionnaires. Each group was compared to women with AIP.

\begin{tabular}{|l|l|l|l|l|l|l|l|l|}
\hline & $<6$ months ago & p-value $^{*}$ & $\mathbf{6 - 1 2}$ months ago & p-value $^{*}$ & $\mathbf{1 2 - 1 8}$ months ago & p-value & $>$ 18 months ago & p-value* $^{*}$ \\
\hline AIP & $1(6 \%)$ & - & $5(29 \%)$ & - & $2(12 \%)$ & - & $9(53 \%)$ & - \\
\hline CD & $(0 \%)$ & 1 & $4(29 \%)$ & 1 & $4(29 \%)$ & 0.37 & $6(42 \%)$ & 0.72 \\
\hline Clinic+CD & $1(6 \%)$ & 1 & $7(44 \%)$ & 0.48 & $4(25 \%)$ & 0.4 & $4(25 \%)$ & 0.16 \\
\hline $\mathrm{EPH} / \mathrm{PPH}$ & $4(18 \%)$ & 0.15 & $6(27 \%)$ & 1 & $1(5 \%)$ & 1 & $11(50 \%)$ & 1 \\
\hline
\end{tabular}

P values compared to AIP, using

Fishers exact test 
Table 2

Median IES-R scores for all groups and number of women in each risk category according to their IES-R score. $\mathrm{P}$ values are calculated

\begin{tabular}{|l|c|c|l|l|l|}
\hline & $\begin{array}{l}\text { Median IES-R } \\
\text { score (range) }\end{array}$ & p-value & $\begin{array}{l}\text { Number of women at } \\
\text { low risk of PTSD }(<24 \\
\text { on IES-R) }\end{array}$ & $\begin{array}{l}\text { Number of women at } \\
\text { moderate risk of PTSD } \\
\mathbf{2 4 - 3 2} \text { on IES-R) }\end{array}$ & $\begin{array}{l}\text { Number of women at } \\
\text { high risk of PTSD (>32 } \\
\text { on IES-R) [p-value }\end{array}$ \\
\hline CD $(\mathrm{n}=14)$ & $1.5(0-47)$ & - & 13 & 0 & $1[-]$ \\
\hline AIP $(\mathrm{n}=17)$ & $19(2-76)$ & 0.001 & 10 & 0 & $7[0.045]$ \\
\hline Clinic+CD $(\mathrm{n}=16)$ & $1.5(0-15)$ & 0.726 & 16 & 0 & $0[0.47]$ \\
\hline EPH/PPH $(\mathrm{n}=22)$ & $28.5(2-70)$ & 0.001 & 13 & 2 & $7[0.12]$ \\
\hline
\end{tabular}

$\mathrm{P}$ values compared to the control group of uncomplicated caesarean delivery (CD) using

Mann Whitney U test

Fishers exact test. 